\title{
A pseudo Lindley distribution and its application
}

\author{
Halim Zeghdoudi* and Sihem Nedjar \\ LaPS Laboratory, Badji-Mokhtar University, Box 12, Annaba, 23000, Algeria
}

Received February 6, 2016; Accepted June 21, 2016

Copyright (C) 2016, Afrika Statistika and Statistics and Probability African Society (SPAS). All rights reserved

\begin{abstract}
In this paper, we introduce a new distribution named as the Pseudo Lindley Distribution $(P S L D)$ as a generalization of the Lindley distribution $(L D)$. A full and detailed description are provided in terms of moments, cumulates, characteristic function, failure, rate function, stochastic ordering, distributions of sums, and parameters estimation. Simulations studies and data driven applications are also reported.

Résumé. Dans cet article, nous introduisons une nouvelle distribution de probability dénommée Pseudo-Lindely-Distribution $(P s L D)$ comme une géné- ralisation de celle de Lindley $(L D)$. Une description complète s'en suit, par rapport aux moments, à la fonction caractéristique, à la function de répartition et de survie, aux ordres stochastiques au sein de la famille. De plus l'estimation des paramètres par la méthode des moments et du maximum de vraisemblance est abordée. Une partie réservée aux simulations et à des applications sur des données réelles montrent la souplesse de cette loi pour expliquer certaines données de survie par rapport à la distribution de Lindley et certaines de ces généralisations.
\end{abstract}

Key words: Lindley distribution; Exponential distribution; Gamma distribution; Stochastic ordering; Maximum-likelihood estimation.

AMS 2010 Mathematics Subject Classification : 60E05; $62 \mathrm{H} 20$.

\section{Introduction}

We are concerned with a two-parameters generalization of the one-parameter Lindley distribution (Lindley , 1958) defined below by its probability density function, depending on

\footnotetext{
* Corresponding author Halim Zeghdoudi : halim.zeghdoudi@univ-annaba.dz Sihem Nedjar: nedjar.sihem@yahoo.com
} 
the parameter $\theta>0$,

$$
f(x ; \theta)=\left\{\begin{array}{cc}
\frac{\theta^{2}(1+x) e^{-\theta x}}{1+\theta} & x>0 \\
0, & \text { otherwise }
\end{array}\right.
$$

This distribution has attracted the interest of many researchers and has been generalized several times by various authors. First, Sankaran (1970) used (1) when the parameter follows a Poison Law to derive their discrete Poisson-Lindley Distribution (PLD) with density function

$$
f_{P L D}(x ; \theta)=\frac{\theta^{2}(x+\theta+2) e^{-\theta x}}{(1+\theta)^{x}}, x=0,1, \ldots
$$

where $\theta>0$ is a positive parameter. Recently, Asgharzadeh et al. (2013), Ghitany et al. (2008a) and Ghitany et al. (2008b) introduced new distributions bounded to 1, called Zero-truncated poisson-Lindley and Pareto Poisson-Lindley distributions. Also, Nedjar and Zeghdoudi (2016) and Zeghdoudi and Nedjar (2016) introduced a new distribution, named gamma-Lindley distribution, based on mixtures of gamma $(2, \theta)$ and one-parameter Lindley distributions.

Since the Lindsley distribution and its derived form we just described depend on one parameter, they may lack of flexibility in statistical modelling of different types of lifetime data. This motivated us to find out generalizations of more parameters that are easily handled. Yet Zakerzadah and Dolati (2010) introduced a generalization of with three parameters. But it happens that their distribution is difficult to use and is not as flexible as one can whish.

The idea of this paper is to use a mixture of ordinary exponential random variables ( $r v$ 's) and $\operatorname{Gamma}(2, \theta)$ ones. The found distribution is characterized through a number of properties concerning its characteristics and parameters : probability density function $(p d f)$, cumulative distribution $(c d f)$, survival and hazard rate functions, moment generating function ( $m g f)$, mean, variance and stochastic orderings. Also relevant plots are given as illustrations.

Moment estimates are also discussed and statistical applications treating goodness of fit are provided.

The paper is organized as follows. In Section 2, we introduce a generalization of the Lindley distribution, that we name as the Pseudo-Lindley distribution ( $P s L D)$ and give immediate properties as the mode. Section 3 is devoted to the study of survival and the hazard functions. In Section 4 we focus on the moments including skewness and kirtosis and stochastic ordering. In Section 5, we are interested in parameters estimation using both the maximum likelyhood and the moment method. In this last section, simulation studies are reported and as well, are provided datadriven applications allowing comparisons between our new law with the original Lindley law and with others of it generalizations. We finish the paper with a conclusive section.

\section{The Pseudo-Lindley distributions and immediate properties}

In this section, we give the pseudo lindley distribution and study its properties. Let $Y_{1} \sim$ $\operatorname{Exp}(\theta)$ and $Y_{2} \sim \Gamma(2, \theta)$ be two independent random variables. For $\beta \geq 1$, we consider the 
mixture random variable variable $X=Y_{1}$ and $X=Y_{2}$ with probability $(\beta-1) / \beta$ and $1 / \beta$ respectively. The density function of $X$ is given by:

$$
f_{P s L D}(x ; \theta, \beta)=\left\{\begin{array}{cl}
\frac{\theta(\beta-1+\theta x) e^{-\theta x}}{\beta}, & x, \theta, \beta \geq 1 \\
0, & \text { otherwise. }
\end{array}\right.
$$

Remark 1. Let us make these two remarks.

(1) If $\beta=\theta+1$, this distribution is lindley distribution.

(2) If $\beta=1$, this distribution is a $\Gamma(2, \theta)$ distribution.

To find the mode, we may see that the first and second derivatives of $f_{P s L D}(x)$ are given by

$$
\frac{d}{d x} f_{P s L D}(x)=\frac{\theta^{2}(2-\beta-\theta x) e^{-\theta x}}{\beta}=0 \text { gives } x=\frac{2-\beta}{\theta} \text { and } \frac{d^{2}}{d x^{2}} f_{P s L D}(\hat{x})<0 .
$$

For $1 \leq \beta<2, \hat{x}=(2-\beta) / \theta$ is the unique critical point which $f_{P s L D}(x ; \theta, \beta)$ is maximum.

For $\beta \geq 2$,

$$
\frac{d}{d x} f_{P s L D}(x ; \theta, \beta) \leq 0,
$$

and then then the density function $f_{P s L D}(x ; \theta, \beta)$ is decreasing in $x$. Therefore, the mode of PsLD is given by

$$
\operatorname{mode}(X)=\left\{\begin{array}{cc}
\frac{2-\beta}{\theta} & \text { for } 1 \leq \beta<2 \\
0 & \text { otherwise }
\end{array}\right.
$$

We can easily find the cumulative distribution function $(c d f)$ of the PsLD :

$$
F_{P s L D}(x)=1-\frac{(\beta+\theta x) e^{-\theta x}}{\beta} ; x, \theta>0>0, \beta \geq 1 .
$$

\section{Pseudo lindley distribution(PsLD) and some properties}

\subsection{Survival and hazard rate function}

These two functions are

$$
S_{P s L D}(x)=1-F_{P s L D}(x)=\frac{(\beta+\theta x) e^{-\theta x}}{\beta}
$$

and

$$
h_{P s L D}(x)=\frac{f_{P s L D}(x)}{1-F_{P s L D}(x)}=\frac{\theta(\beta+\theta x-1)}{\beta+\theta x}
$$

be the survival and hazard rate function, respectively.

Proposition 1. Let $h_{P s L D}(x)$ be the hazard rate function of $X$. Then $h_{P s L}(x)$ is increasing. 
H. Zeghdoudi and S. Nedjar, Afrika Statistika, Vol. 11(1), 2016, pages 923-932. A pseudo Lindley distribution and its application.

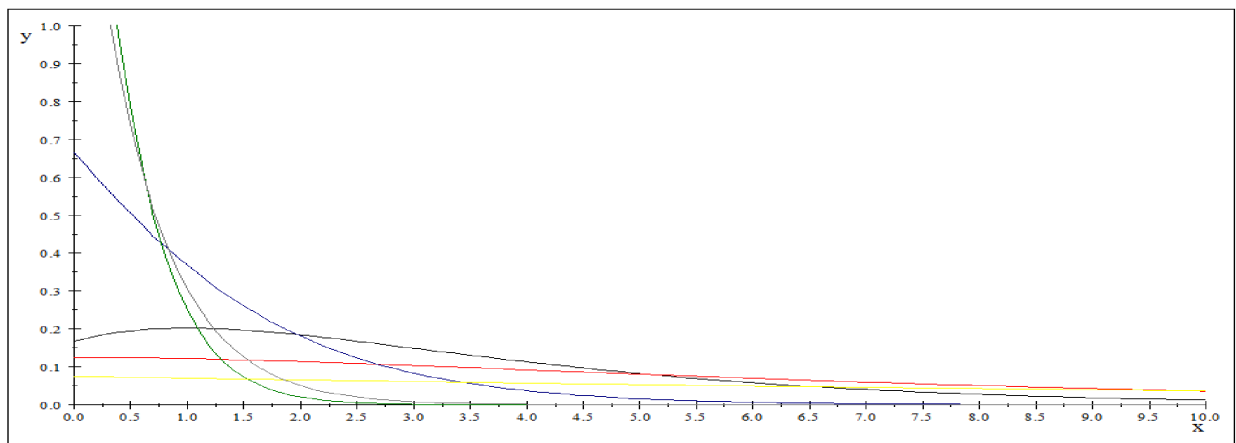

Fig. 1. Plots of the density function for some parameter values, black $(0.5,1.5)$; $\operatorname{red}(0.25,2)$; bleu(1,3); green $(3,3)$; yellow $(0.1,4)$; gray $(2,8)$

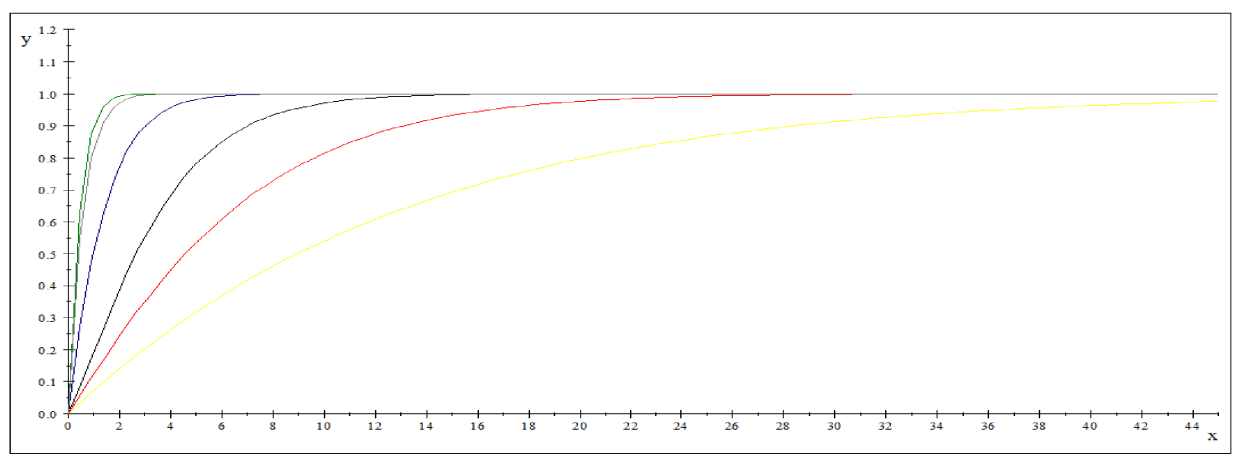

Fig. 2. Plots of the distribution function for some parameter values, black $(0.5,1.5)$; $\operatorname{red}(0.25,2)$; bleu $(1,3)$; green $(3,3)$; yellow $(0.1,4)$; $\operatorname{gray}(2,8)$

Proof. It easy to check that

$$
\frac{d}{d x} h_{P s L D}(x)=\frac{\theta^{2}}{(\beta+\theta x)^{2}}>0 .
$$

\section{Moments and related measures}

The $k t h$ moment about the origin of the PsLD is :

$$
\mathbb{E}\left(X^{k}\right)=\frac{k !(\beta+k)}{\theta^{k} \beta}, k=1,2, \ldots
$$

Proposition 2. Let $X_{1}, X_{2}, \ldots, X_{n}$ be $n$ independent random variables from $\operatorname{PsLD}(\beta, \theta)$ distribution. Then the moment generating function(mgf) of $S=\sum_{i=1}^{n} X_{i}$, is given by

$$
M_{S}(t)=\frac{\theta^{n}((1-\beta) t+\theta \beta)^{n}}{\beta^{n}(t-\theta)^{2 n}}
$$


H. Zeghdoudi and S. Nedjar, Afrika Statistika, Vol. 11(1), 2016, pages 923-932. A pseudo Lindley distribution and its application.

and

$$
M_{X}(t)=\mathbb{E}\left(e^{t X}\right)=\frac{\theta((1-\beta) t+\theta \beta)}{\beta(t-\theta)^{2}}
$$

Remark 2. The kth moment about the origin of the exponential distribution is

$$
\mathbb{E}\left(X^{k}\right)=\frac{k !}{\theta^{k}}
$$

Furthermore, the moment generating function of $X$ and $S$ exists, that is $\mathbb{E} e^{t X}<+\infty$, if $t<\theta$.

Corollary 1. Let $X \sim P \operatorname{PLD}(\beta, \theta)$, the mean and variance of $X$ are :

$$
\mathbb{E}(X)=\frac{\beta+1}{\theta \beta}, \operatorname{Var}(X)=\frac{\beta^{2}+2 \beta-1}{\beta^{2} \theta^{2}}
$$

Theorem 1. Let $X \sim P s L D(\beta, \theta), M=\operatorname{mode}(X)$, me $=\operatorname{median}(X)$ and $\mu=E(X)$. Then $M<m e<\mu$.

Proof. According to the increasingness of $F(x)$ for all $x, \theta$ and $\beta$,

$$
F(M)=\left\{\begin{array}{cc}
1-\frac{2 e^{-(2-\beta)}}{\beta} & \text { for } 1 \leq \beta<2 \\
0 & \text { otherwise }
\end{array}, F(m e)=\frac{1}{2}\right.
$$

and

$$
F(\mu)=1-\frac{\left(\beta^{2}+\beta+1\right) e^{-\left(\frac{\beta+1}{\beta}\right)}}{\beta^{2}}
$$

Note that $F(M)$ is a decreasing function for all $\beta \geq 1$. It easy to check that $F(M)<$ $F(m e)<F(\mu)$.To this end, we have $M<m e<\mu$.

The coefficients of variation $\gamma$, skewness and kurtosis of the PsLD are obtained as follows

$$
\begin{aligned}
\gamma & =\frac{\sqrt{\operatorname{Var}(X)}}{\mathbb{E}(X)}=\frac{\sqrt{\beta^{2}+2 \beta-1}}{\beta+1} \\
\text { skewness } & =\frac{\mathbb{E}\left(X^{3}\right)}{(\operatorname{Var}(X))^{\frac{3}{2}}}=\frac{6 \beta^{2}(\beta+3)}{\left(\beta^{2}+2 \beta-1\right)^{\frac{3}{2}}} \\
\text { kurtosis } & =\frac{\mathbb{E}\left(X^{4}\right)}{(\operatorname{Var}(X))^{2}}=\frac{24 \beta^{3}(\beta+4)}{\left(\beta^{2}+2 \beta-1\right)^{2}}
\end{aligned}
$$

Remark 3. All these expressions are independent of the parameter $\theta$ and depend upon the parameter $\beta$ only.

Journal home page: www.jafristat.net 
H. Zeghdoudi and S. Nedjar, Afrika Statistika, Vol. 11(1), 2016, pages 923-932. A pseudo Lindley distribution and its application.

\subsection{Stochastic orders}

Definition 1. Consider two random variables $X$ and $Y$. We define these four stochastic orders.

(a) $X$ is said to be smaller than $Y$ in stochastic order, denoted

$$
X \prec_{s} Y,
$$

if and only if

$$
F_{X}(t) \geq F_{Y}(t), \text { for all } t
$$

b) $X$ is said to be smaller than $Y$ in convex order, denoted

$$
X \leq_{c x} Y
$$

if for any convex function $\phi$ and provided expectations exist,

$$
E[\phi(X)] \leq E[\phi(Y)]
$$

c) $X$ is said to be smaller than $Y$ in hazard rate order, denoted

$$
X \prec_{h r} Y
$$

if and only if

$$
h_{X}(t) \geq h_{Y}(t), \text { for all } t
$$

(d) $X$ is said smaller than $Y$ in likelihood ratio order, denoted

$$
X \prec_{l r} Y,
$$

if and only if $f_{X}(t) / f_{Y}(t)$ is decreasing in $t$.

Remark 4. Likelihood ratio order $\Rightarrow$ Hazard rate order $\Rightarrow$ Stochastic order.

If $E[X]=E[Y]$, then Convex order $\Leftrightarrow$ Stochastic order.

Theorem 2. Let $X_{i} \sim P s L D\left(\beta_{i}, \theta_{i}\right), i=1,2$ be two random variables. If $\theta_{1}=\theta_{2}$ and $\beta_{1} \geq \beta_{2}$, then $X_{1} \prec_{l r} X_{2}, X_{1} \prec_{h r} X_{2}, X_{1} \prec_{s} X_{2}$ and $X_{1} \leq_{c x} X_{2}$.

Proof. We have

$$
\frac{f_{X_{1}}(t)}{f_{X_{2}}(t)}=\frac{\theta_{1} \beta_{2}\left(\beta_{1}-1+\theta_{1} t\right)}{\theta_{2} \beta_{1}\left(\beta_{2}-1+\theta_{2} t\right)} e^{-\left(\theta_{1}-\theta_{2}\right) t}
$$

For simplicity's sake, we use $\ln \left(\frac{f_{X_{1}}(t)}{f_{X_{2}}(t)}\right)$. Now, we can find

$$
\frac{d}{d t} \ln \left(\frac{f_{X_{1}}(t)}{f_{X_{2}}(t)}\right)=-\left(\theta_{1}-\theta_{2}\right)+\frac{\theta_{1}\left(\beta_{2}-1\right)-\theta_{2}\left(\beta_{1}-1\right)}{\left(\beta_{1}-1+\theta_{1} t\right)\left(\beta_{2}-1+\theta_{2} t\right)}
$$

To this end, if $\theta_{1}=\theta_{2}$ and $\beta_{1} \geq \beta_{2}$, we have $\frac{d}{d t} \ln \left(\frac{f_{X_{1}}(t)}{f_{X_{2}}(t)}\right) \leq 0$. This means that $X_{1} \prec_{l r} X_{2}$. Also, according to Remark 4 , the theorem is proved.

Journal home page: www.jafristat.net 
H. Zeghdoudi and S. Nedjar, Afrika Statistika, Vol. 11(1), 2016, pages 923-932. A pseudo Lindley distribution and its application.

\section{Estimation of parameters}

5.1. Maximum Likelihood Estimates (MLE)

Let $X_{i} \sim P \operatorname{PL} D(\beta, \theta), i=\overline{1, n}$ be $n$ random variables. The ln-likelihood function, $\ln l\left(x_{i} ; \beta, \theta\right)$ is:

$$
\ln l\left(x_{i} ; \beta, \theta\right)=n \ln \theta-n \ln \beta+\sum_{i=1}^{n} \ln \left(\beta-1+\theta x_{i}\right)-\theta \sum_{i=1}^{n} x_{i} .
$$

The derivatives of $\operatorname{Lnl}\left(x_{i} ; \beta, \theta\right)$ with respect to $\theta$ and $\beta$ are:

$$
\begin{gathered}
\frac{\partial \ln l\left(x_{i} ; \beta, \theta\right)}{\partial \theta}=\frac{n}{\theta}-\sum_{i=1}^{n} x_{i}+\sum_{i=1}^{n}\left(\frac{x_{i}}{\beta-1+\theta x_{i}}\right) \\
\frac{\partial \ln l\left(x_{i} ; \beta, \theta\right)}{\partial \beta} 0=\frac{-n}{\beta}+\sum_{i=1}^{n}\left(\frac{1}{\beta-1+\theta x_{i}}\right)
\end{gathered}
$$

The two equations (6) and (7) cannot be solved directly, we must used the Fisher scoring method. We have

$$
\left[\begin{array}{ll}
\frac{\partial^{2} \operatorname{Lnl}\left(x_{i} ; \beta, \theta\right)}{\partial \theta^{2}} & \frac{\partial^{2} \operatorname{Lnl}\left(x_{i} ; \beta, \theta\right)}{\partial \theta \partial \beta} \\
\frac{\partial^{2} \operatorname{Lnl}\left(x_{i} ; \beta, \theta\right)}{\partial \beta \partial \theta} & \frac{\partial^{2} \operatorname{Lnl}\left(x_{i} ; \beta, \theta\right)}{\partial \beta^{2}}
\end{array}\right]_{\substack{\hat{\theta}=\theta_{0} \\
\hat{\beta}=\beta_{0}}}\left[\begin{array}{c}
\hat{\theta}-\theta_{0} \\
\hat{\beta}-\beta_{0}
\end{array}\right]=\left[\begin{array}{c}
\frac{\partial L n l\left(x_{i} ; \beta, \theta\right)}{\partial \theta} \\
\frac{\partial \operatorname{Lnl}\left(x_{i} ; \beta, \theta\right)}{\partial \beta}
\end{array}\right]_{\begin{array}{c}
\hat{\theta}=\theta_{0} \\
\hat{\beta}=\beta_{0}
\end{array}}
$$

where, $\frac{\partial^{2} \operatorname{Lnl}\left(x_{i} ; \beta, \theta\right)}{\partial \theta^{2}}=-\frac{n}{\theta^{2}}-\sum_{i=1}^{n}\left(\frac{x_{i}^{2}}{\left(\beta-1+\theta x_{i}\right)^{2}}\right) ; \frac{\partial^{2} \operatorname{Lnl}\left(x_{i} ; \beta, \theta\right)}{\partial \beta^{2}}=\frac{n}{\beta^{2}}-\sum_{i=1}^{n}\left(\frac{1}{\left(\beta-1+\theta x_{i}\right)^{2}}\right)$ and $\frac{\partial^{2} \operatorname{Lnl}\left(x_{i} ; \beta, \theta\right)}{\partial \beta \partial \theta}=\frac{\partial^{2} \operatorname{Lnl}\left(x_{i} ; \beta, \theta\right)}{\partial \theta \partial \beta}=-\sum_{i=1}^{n}\left(\frac{x_{i}}{\left(\beta-1+\theta x_{i}\right)^{2}}\right)$.

The equation (8) can be solved iteratively where $\theta_{0}, \beta_{0}$ are the initial values of $\theta, \beta$.

\subsection{Moments estimates}

Using the first moment $m$ and the variance $s^{2}$ about PsLD, we have

$$
\left\{\begin{array}{c}
m=\frac{\beta+1}{\theta \beta} \\
s^{2}=\frac{\beta^{2}+2 \beta-1}{\theta^{2} \beta^{2}}
\end{array}\right.
$$

We solve this nonlinear system we find the couple $(\hat{\theta}, \hat{\beta})$, where $(\hat{\theta}, \hat{\beta})>0$ for $s>0, m>0$.

$$
\hat{\theta}=\frac{2 m+\sqrt{2} \sqrt{m^{2}-s^{2}}}{m^{2}+s^{2}} \text { et } \hat{\beta}=\frac{m^{2}+s^{2}}{m^{2}-s^{2}+\sqrt{2} m \sqrt{m^{2}-s^{2}}}
$$

Theorem 3. The estimator $(M M) \hat{\theta}$ of $\theta$ is positively biased i.e, $E(\hat{\theta})>\theta$ 
Proof.Let $\hat{\theta}=N(m)$ and $N(t)=\frac{\beta+1}{t \beta}$, for $t>0$ we have for $t$ and fixed $\beta$

$$
\frac{d^{2}}{d t^{2}} N(t)=\frac{2(\beta+1)}{\beta t^{3}}>0
$$

$N(t)$ is strictly convex. Now, by Jensen's inequality, we have $\mathbb{E}(N(m))>N(\mathbb{E}(m))$.

Thus, $\mathbb{E}(m)=N(\mu)=N\left(\frac{\beta+1}{\theta \beta}\right)=\theta, E(\hat{\theta})>\theta$. Then $\hat{\theta}$ is positively biased.

Theorem 4. The estimator(MM) $\hat{\beta}$ of $\beta$ is positively biased.

Proof. Let $\hat{\beta}=g(m)$ and $g(t)=\frac{1}{\theta t-1}$, for $t>0$ we have for $t$ and fixed $\theta$

$$
\frac{d^{2}}{d t^{2}} g(t)=\frac{2 \theta^{2}}{(\theta t-1)^{3}}>0
$$

$g(t)$ is strictly convex. Now, by Jensen's inequality, we have $\mathbb{E}(g(m))>g(\mathbb{E}(m))$.

Thus, $\mathbb{E}(m)=g(\mu)=g\left(\frac{\beta+1}{\theta \beta}\right)=\beta, E(\hat{\beta})>\beta$. Then $\hat{\beta}$ is positively biased.

\subsection{Illustrative examples}

Example 1. In this section, we give some simulation for four series of moments estimator parameters distribution which Lindley, PL, Exponential and Gamma distribution. For the exponential distribution; $\hat{\theta}=\frac{1}{m}$, Lindley distribution; $\hat{\theta}=\frac{-(m-1)+\sqrt{(m-1)^{2}+8 m}}{2 m}$ and gamma distribution; $\hat{\theta}=\frac{s^{2}}{m}, \hat{\alpha}=\frac{m^{2}}{s^{2}}$, see Tables 1 and 2 .

\begin{tabular}{|l|l|l|l|}
\hline $\operatorname{Distr}(L D)$ & $m$ & $s$ & $\hat{\theta}$ \\
\hline Serie1 & 100 & 71 & 0.0198 \\
\hline Serie2 & 50 & 36 & 0.0392 \\
\hline Serie3 & 15 & 12 & 0.1258 \\
\hline Serie4 & 3.32 & 2.34 & 0.5017 \\
\hline
\end{tabular}

\begin{tabular}{|l|l|l|l|l|}
\hline $\operatorname{Distr}(P s L D)$ & $m$ & $s$ & $\hat{\theta}$ & $\hat{\beta}$ \\
\hline Serie1 & 100 & 71 & 0.01998 & 1.0083 \\
\hline Serie2 & 50 & 36 & 0.03927 & 1.0378 \\
\hline Serie3 & 15 & 12 & 0.11579 & 1.357 \\
\hline Serie4 & 3.32 & 2.34 & 0.60436 & 1.003 \\
\hline
\end{tabular}

Table 1.

\begin{tabular}{|l|l|l|l|}
\hline $\operatorname{Distr}(\operatorname{Exp})$ & $m$ & $s$ & $\hat{\theta}$ \\
\hline Serie1 & 100 & 71 & 0.0100 \\
\hline Serie2 & 50 & 36 & 0.0200 \\
\hline Serie3 & 15 & 12 & 0.0666 \\
\hline Serie4 & 3.32 & 2.34 & 0.3012 \\
\hline
\end{tabular}

\begin{tabular}{|l|l|l|l|l|}
\hline Distr(Gamma) & $m$ & $s$ & $\hat{\theta}$ & $\hat{\alpha}$ \\
\hline Serie1 & 100 & 71 & 50.41 & 1.9837 \\
\hline Serie2 & 50 & 36 & 25.92 & 1.9290 \\
\hline Serie3 & 15 & 12 & 9.6 & 1.5625 \\
\hline Serie4 & 3.32 & 2.34 & 1.6493 & 2.013 \\
\hline
\end{tabular}

Table 2 .

Now, we used Data of survival times (in months) of 94 guinea individus infected with Ebola virus, see Table 3 . 
H. Zeghdoudi and S. Nedjar, Afrika Statistika, Vol. 11(1), 2016, pages 923-932. A pseudo Lindley distribution and its application.

\begin{tabular}{|l|l|l|l|}
\hline Survival time $m=3.17, s=2.095$ & Obsf req & LD $\hat{\theta}=0.522$ & $P s L D \hat{\theta}=0.772, \hat{\beta}=1.004$ \\
\hline$[0,2[$ & 32 & 38.217 & 32.651 \\
\hline$[2,4[$ & 35 & 28.16 & 35.981 \\
\hline$[4,6[$ & 17 & 15.089 & 16.37 \\
\hline$[6,8[$ & 7 & 7.33 & 6.0458 \\
\hline$[8,10]$ & 3 & 3.152 & 2.0285 \\
\hline Total & 94 & 94 & 94 \\
\hline$\chi^{2}$ & - & 2.9244 & 0.6796 \\
\hline
\end{tabular}

Table 3.

Example 2. We consider from Lawless (2003), pp. 204 and 263 two series of real data. The first one, represents the failure times $(\mathrm{mm})$ for a sample of fifteen electronic components in an acceleration life test : $1.4,5.1,6.3,10.8,12.1,18.5,19.7,22.2,23,30.6,37.3,46.3$, $53.9,59.8,66.2$. The second set of data, are the number of cycles to failure for $25100-\mathrm{cm}$ specimens of yarn, tested at a particular strain level : 15, 20, 38, 42, 61, 76, 86, 98, 121, 146, 149, 157, 175, 176, 180, 180, 198, 220, 224, 251, 264, 282, 321, 325, 653, see Table 4.

\begin{tabular}{|l|l|l|l|l|l|}
\hline \multirow{2}{*}{$\begin{array}{l}\text { Data } \\
\text { Serie } 1\end{array}$} & Distribution & $\beta$ & $\theta$ & log-likelihood & Kolmogrov-Smirnov \\
\cline { 2 - 6 } & PsLD & 1.129 & 0.684 & -62.075 & 0.82 \\
\cline { 2 - 6 }$m=27.546$ & Gamma & 1.442 & 0.052 & -64.197 & 0.102 \\
\cline { 2 - 6 } & Weibull & 1.306 & 0.034 & -64.026 & 0.450 \\
\hline$s=20.059$ & Lognormal & 1.061 & 2.931 & -65.626 & 0.163 \\
\hline \multirow{2}{*}{ Serie 2} & PsLD & 1.086 & 0.010 & -150.232 & 0.128 \\
\hline$n=25$ & Gamma & 1.794 & 0.010 & -152.371 & 0.135 \\
\hline$m=178.32$ & Weibull & 1.414 & 0.005 & -152.440 & 0.697 \\
\hline$s=131.097$ & Lognormal & 0.891 & 4.880 & -154.092 & 0.155 \\
\hline
\end{tabular}

Table 4.

\section{Conclusion}

In this work, we proposed a two parameter $P S L D$, of which the $L D$ is a particular case. Several properties have been discussed : moments, cumulates, characteristic function, failure rate function, stochastic ordering, distributions of sums, the maximum likelihood method and the method of moments. The $L D$ does not provide enough flexibility for analyzing and modeling different types of lifetime data and survival analysis. But the PSLD is flexible , simple and easy to handle. Many properties and applications are given which confirm the goodness of fit and it is better than Lindley, Exponential, Gamma, Weibull, Lognormal distributions.

\section{Acknowledgements}

The authors are grateful for the comments and suggestions by the referee and the Editor. Their comments and suggestions greatly improved the article. 
H. Zeghdoudi and S. Nedjar, Afrika Statistika, Vol. 11(1), 2016, pages 923-932. A pseudo Lindley distribution and its application.

\section{References}

Asgharzadeh, A., Bakouch, Hassan, S. and Esmaeili, L., 2013. Pareto Poisson-Lindley distribution with applications. J. Appl. Stat. 40(8), 1717-1734.

Ghitany, M. E., Al-Mutairi, D.K. and Nadarajah,S., 2008. Zero-truncated Poisson-Lindley distribution and its application, Math. Comput. Simulation 79, 279-287.

Ghitany, M. E., Atieh, B. and Nadarajah, S., 2008. Lindley distribution and its applications. Mathematics and Computers in Simulation, 78, 493-506.

Lawless, J., 2003. Statistical models and methods for lifetime data. Wiley, New York.

Lindley, D. V., 1958. Fiducial distributions and Bayes'theorem. Journal of the Royal Society, series B, 20, 102-107.

Nedjar, S. and Zeghdoudi, H., 2016. Gamma Lindley distribution and its application. Journal of Applied Probability and Statistics, 11(1), 129-138.

Sankaran, M., 1970. The discrete Poisson-Lindley distribution. Biometrics, 26, 145-149.

Shanker, R. and Sharma, S., 2013. A two-parameter Lindley distribution for modeling waiting and survival times data. Applied Mathematics, 4, 363-368.

Zakerzadah, H. and Dolati, A., 2010. Generalized Lindley distribution. J. Math. Ext, 3(2), $13-25$.

Zeghdoudi, H. and Nedjar, S., 2016. On gamma Lindley distribution : Properties and Simulations. Journal of Computational and Applied Mathematics, 298, 167-174. 